\title{
ON THE EXISTENCE OF GREEN'S FUNCTION IN RIEMANNIAN MANIFOLDS
}

\author{
JOSE L. FERNÁNDEZ
}

\begin{abstract}
This note provides a sufficient condition of geometric character for the existence of Green's function in an arbitrary complete Riemannian manifold.
\end{abstract}

1. The purpose of this note is to present a sufficient condition for an arbitrary complete $C^{\infty}$ Riemannian manifold $M$ to possess Green's function.

The condition is given in terms of some isoperimetric inequalities which we now describe.

Define $\varphi_{M}(t)$ by

$$
\varphi_{M}(t)=\inf \left\{\begin{array}{ll}
A(\partial \Omega): & \Omega, \text { smooth relatively compact domain } \\
& \text { in } M \text { with volume } \geqslant t,
\end{array}\right\}
$$

where $A$ denotes the $((\operatorname{dim} M)-1)$-dimensional measure induced by the metric of $M$. In particular $\varphi_{M}(V(\Omega)) \leqslant A(\partial \Omega)$ for each such $\Omega$.

In $§ 2$ we will prove:

THEOREM. If $\int V(M) d t / \varphi_{M}(t)^{2}<\infty$, then $M$ has a Green's function.

Recently, Dodziuk has proved in [D] that if $\varphi_{M}(t) \geqslant c t$, i.e. if the isoperimetric inequality holds in $M$, then $M$ has a Green's function (see also [T, p. 438]).

In [V2], Varopoulos has shown by extending a classical result of Ahlfors [A], that if we let $L(t)=A\left(\partial B\left(x_{0}, t\right)\right)$ for a point $x_{0} \in M$, where $B\left(x_{0}, t\right)$ denotes the ball around $x_{0}$ of radius $t$, then $\int^{\infty} d t / L(t)<\infty$ if $M$ has a Green's function. To see the relation with the theorem above define $\tilde{\varphi}_{M}$ by taking the infimum only on balls around $x_{0}$, i.e.

$$
\tilde{\varphi}_{M}\left(V\left(B\left(x_{0}, r\right)\right)\right)=A\left(\partial B\left(x_{0}, r\right)\right) .
$$

But then, since $(d / d r) V\left(B\left(x_{0}, r\right)\right)=A\left(\partial B\left(x_{0}, r\right)\right)$, we have that

$$
\int^{V(M)} \frac{d t}{\tilde{\varphi}_{M}(t)^{2}}=\int^{\infty} \frac{d V\left(B\left(x_{0}, t\right)\right)}{\tilde{\varphi}_{M}\left(V\left(B\left(x_{0}, t\right)\right)\right)^{2}}=\int^{\infty} \frac{d t}{L(t)} .
$$

Thus our sufficient condition is close to the Ahlfors-Varopoulos necessary condition.

We should remark that, as shown in [V1], the latter is also sufficient if the Ricci curvature is (semi-)positive definite but not in general; and that Milnor [M] (see also [GW]) showed that it is necessary and sufficient for 2-dimensional models.

Received by the editors November 19, 1984 and, in revised form, February 26, 1985.

1980 Mathematics Subject Classification. Primary 31C12; Secondary 30F20.

(C)1986 American Mathematical Society $0002-9939 / 86 \$ 1.00+\$ .25$ per page 
Since in models the Laplacian is also rotationally invariant around the pole, one can check, following the proof of the theorem, that, for $n$-dimensional models, $\int^{\infty} d t / L(t)<\infty$ is necessary and 'sufficient' for the existence of Green's function.

2. Proof. Assume that there is no Green's function. Let $\left\{\Omega_{n}\right\}_{n=0}^{\infty}$ be a sequence of smooth domains in $M$ such that $\bar{\Omega}_{n} \subset \Omega_{n+1}, \cup_{n=0}^{\infty} \Omega_{n}=M$ and $\Omega_{0}$ is a ball so small that $\left.A\left(\partial \Omega_{0}\right) \leqslant \frac{1}{2} \varphi_{M}\left(V \backslash \Omega_{0}\right)\right)$. Let $\omega_{n}$ be the solution to the Dirichlet problem in $\Omega_{n} \backslash \bar{\Omega}_{n}$ with data $\omega_{n}=0$ on $\partial \Omega_{0}, \omega_{n}=1$ on $\partial \Omega_{n}$.

Since we are assuming that there is no Green's function we have that $\omega_{n} \downarrow 0$ uniformly on compact subsets of $M \backslash \bar{\Omega}_{0}$. Moreover, if $N$ denotes the outer normal vector field on $\partial \Omega_{0}$, then $0<d \omega_{n}(N) \downarrow 0$.

If $\|\cdot\|$ denotes the norm in $T M$, then by the divergence theorem and since $\omega_{n}$ is harmonic we have, for $0 \leqslant \delta<\varepsilon<1$,

$$
\int_{\delta<\omega_{n}<\varepsilon}\left\|\nabla \omega_{n}\right\|^{2} d V=(\varepsilon-\delta) \int_{\partial \Omega_{0}} \nabla \omega_{n} \cdot N d A
$$

By the coarea formula, see [F], we have

$$
\int_{\delta<\omega_{n}<\varepsilon}\left\|\nabla \omega_{n}\right\| d V=\int_{\delta}^{\varepsilon} A\left(\omega_{n}=t\right) d t
$$

Setting $\alpha_{n}=\int_{\partial \Omega_{0}} \nabla \omega_{n} \cdot N d A$ and using the Schwartz inequality, (1) and (2), we obtain

$$
\left[\int_{\delta}^{\varepsilon} A\left(\omega_{n}=t\right) d t\right]^{2} \leqslant(\varepsilon-\delta) \alpha_{n} \cdot V\left(\delta<\omega_{n}<\varepsilon\right)
$$

Therefore

$$
\left[\frac{1}{\varepsilon-\delta} \int_{\delta}^{\varepsilon} A\left(\omega_{n}=t\right) d t\right]^{2} \leqslant \alpha_{n} \frac{V\left(\delta<\omega_{n}<\varepsilon\right)}{\varepsilon-\delta}
$$

and, if $V_{n}(\varepsilon)=V\left(0<\omega_{n}<\varepsilon\right)$, we have that

$$
A\left(\omega_{n}=\varepsilon\right)^{2} \leqslant \alpha_{n} \frac{d}{d \varepsilon} V_{n}(\varepsilon) .
$$

Since, by assumption, $A\left(\omega_{n}=\varepsilon\right)+A\left(\omega_{n}=0\right) \geqslant \varphi_{M}(V(\varepsilon))$ we obtain

$$
\left[\varphi_{M}\left(V_{n}(\varepsilon)\right)-A\left(\omega_{n}=0\right)\right]^{2} \leqslant \alpha_{n} V_{n}^{\prime}(\varepsilon)
$$

But $\omega_{n} \downarrow 0$ uniformly on compact subsets of $M \backslash \bar{\Omega}_{0}$ which implies that $V_{n}(1 / 2) \uparrow V\left(M \backslash \bar{\Omega}_{0}\right)$. Thus since $\varphi_{M}\left(V_{n}(1 / 2)\right)>\frac{1}{2} A\left(\partial \Omega_{0}\right)$, we obtain

$$
\frac{1}{4} \varphi\left(V_{n}(\varepsilon)\right)^{2} \leqslant \alpha_{n} V_{n}^{\prime}(\varepsilon) \text { for } \varepsilon>\frac{1}{2}, n>n_{0}
$$

for some $n_{0} \in \mathbf{N}$. Therefore,

$$
\frac{1}{8} \leqslant \alpha_{n} \int_{1 / 2}^{1} \frac{V_{n}^{\prime}(\varepsilon) d \varepsilon}{\varphi_{M}\left(V_{n}(\varepsilon)\right)^{2}} \leqslant \alpha_{n} \int_{V_{1}(1 / 2)}^{V(M)} \frac{d t}{\varphi(t)^{2}}
$$

which contradicts the fact that $\alpha_{n} \rightarrow 0$, and so completes the proof. 


\section{REFERENCES}

[A] L. V. Ahlfors, Sur le type d'une surface de Riemann, C. R. Acad. Sci. Paris 201 (1935), 30-32.

[D] J. Dodziuk, Every covering of a compact Riemannian surface of genus greater that one carries a nontrivial $L^{2}$ harmonic differential, Acta Math. 152 (1984), 49-56.

[F] H. Federer, Geometric measure theory, Springer-Verlag, New York, 1969.

[GW] R. Greene and H. Wu, Function theory on manifolds which possess a pole, Lecture Notes in Math., vol. 699, Springer-Verlag, Berlin and New York, 1979.

[M] J. Milnor, On deciding whether a surface is parabolic or hyperbolic, Amer. Math. Monthly 84 (1977), 43-46.

[T] M. Tsuji, Potential theory in modern function theory, Chelsea, New York, 1973.

[V1] N. T. Varopoulos, The Poisson kernel on positively curved manifolds, J. Funct. Anal. 44 (1981), $109-118$.

[V2] _ Potential theory and diffusion on Riemannian manifolds, Conference in Harmonic Analysis in Honor of Antoni Zygmund, Wadsworth, Belmont, Calif. 1983.

Department of Mathematics, University of Wisconsin, Madison, Wisconsin 53706 